\title{
Experimental validation of minimum cost function-based model predictive converter control with efficient reference tracking
}

\author{
Muslem Uddin', Saad Mekhilef', Marco Rivera² \\ ${ }^{1}$ Power Electronics and Renewable Energy Research Laboratory (PEARL), Department of Electrical Engineering, \\ University of Malaya, 50603 Kuala Lumpur, Malaysia \\ ${ }^{2}$ Department of Industrial Technologies, Universidad de Talca, Curico, Chile \\ E-mail:saad@um.edu.my
}

\begin{abstract}
This study proposes a robust and powerful finite control set-model predictive control (MPC) algorithm to control the load current with lower total harmonic distortion and efficient reference tracking. In this control, the cost functions are determined for all the possible switching states of the converter and a switching state is selected corresponding to the minimum cost function for actuating the converter in the next sampling time period. To justify the performance of the proposed MPC scheme, a comprehensive study with the carrier-based pulse-width modulation, hysteresis current control and proposed minimum cost function-based MPC of the three-phase load current has been verified in MATLAB Simulink as well as the validation with dSPACE RTI1 104 experimentation. This study validates the robustness of the proposed minimum cost function-based MPC control with a RL-load and three-phase induction motor (IM) load. The simulation and experimental results justifies the proposed MPC algorithm with potential tracking of the RL-load current corresponding to the reference current with lower harmonic contents as well as tracking of predictive torque and flux compared to the nominal torque and reference flux of IM, respectively.
\end{abstract}

\section{Introduction}

The current control of inductive load with lower harmonic is the prime issue that earns a great concern to power electronic research. Therefore the attention has been dedicated to finite control set MPC control because of the outstanding development of the fast and powerful microprocessors and nowadays it is available to implement the predictive control algorithm as it requires higher number of calculations comparing with all the classical control methods stated in [1]. The proposed algorithm is based on the prediction of the future system behaviour of the load by utilising a discrete-time domain model of the system and can apply to any converter. Different control methods have been focused under this proposed predictive control method. Till date different methods of the predictive control have been investigated and applied to power converters such as finite control set-model predictive control (MPC) [2,3], dead beat control [4] and generalised model predictive control [5]. Recently, MPC current control for different converters such as voltage source inverter, active front end rectifier and matrix converter have been successfully investigated in [6-8], respectively. Furthermore, in [9], a new approach to the control problem of switch-mode single-phase rectifiers has been implemented which is based on a particular MPC algorithm incorporated with soft constraints of key control targets. In direct matrix converter, a method of source

278

(c) The Institution of Engineering and Technology 2015 current reference generation for the predictive control method have been proposed in [10] based on instantaneous active power, the output current reference and system parameters. In [11], based on space vector modulation an improved predictive current control method was implemented for grid-connected voltage source converter during unbalanced network in the positive synchronous reference frame without current-sequential decomposition. In the literature, a digital control method assumes with a reference input voltage vector and aims to approximate it to the nearest inverter vector have been proposed for three-phase-three-stage hybrid multilevel inverter in [1214]. A time-delay compensation has been considered to improve the control performance of the system and an improved digital peak current (IDPC) predictive control technique for switching $\mathrm{dc}-\mathrm{dc}$ converters was then proposed and studied in [15]. Also, for the improvement of the control performance, the predictive control has been applied to a three-phase four-leg inverter with time-delay compensation in [16]. An average sliding control method have been proposed and applied to power factor correction circuits for minimisation of total harmonic distortion (THD) from the input current by suppressing the input current harmonics in [17]. Some recent applications have been investigated to reduce the common mode voltage in three-phase inverters-fed AC drive systems [18] and for rejection of harmonics from the system in [19-21]. 
A complete review for the improvement of input current as well as THD\% analysis in both the input and output side of the indirect matrix converter for predictive control algorithm was investigated in [22].

In the cost function of the MPC method, weighting factor selection is not necessary for the variables with same nature but for the variables with different order of magnitude and unit, the weighting factor selection become as a great issue [23] and the system performance greatly depends on it. In this investigation, current, torque and flux are the controlled variables that need to be controlled. Therefore for induction motor (IM) control it is needed to set the weighting factor but in the case of RL-load no need to set the weighting factor. As in this case controlled variable is only the load current (same nature) but when IM is used as a load then torque and flux (different order and magnitude) need to be controlled which necessitate to set the proper weighting factor $[3,23]$ in the cost function. To overcome this problem, a multi-objective ranking-based predictive IM control has been investigated and this strategy replaces the single cost function with a multi-objective optimisation based on a ranking approach [24]. Recently, a new weighting factor optimisation method has been investigated and applied in the cost function of MPC control algorithm for reduction of torque ripple of IM fed by indirect matrix converter in $[25,26]$ as well as improve the system performance.

This paper proposes a minimum cost function-based MPC algorithm and its application for current control with lower harmonic content and efficient reference tracking which has been compared with the performance of classical control methods in both simulation and experimentation. Also, to validate the robustness of the proposed control method, IM has been used as a load to control the torque and flux with tracking responses.

This paper is organised in the following manner: the power converter topology with inductive load are modelled and presented in Section 2, different classical control philosophies are explained in Section 3, a detailed explanation of MPC control scheme with algorithm including the predictive current, predictive torque-flux and the cost function for the selection of switching state are depicted in Section 4, the simulation and experimental results are discussed in Section 5 and finally, a comprehensive conclusion is drawn in Section 6.

\section{Modelling of the converter}

The converter topology and finite control set switching states for the three-phase VSI are presented in Fig. 1 and Table 1, respectively.

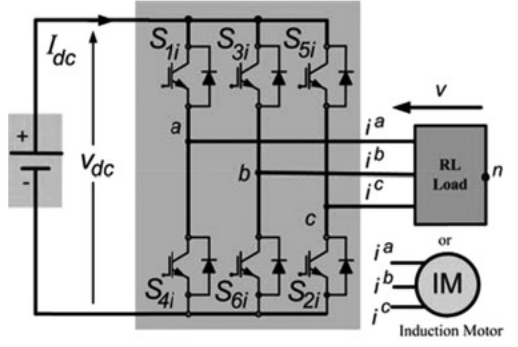

Fig. 1 Converter topology with different load

\subsection{Converter model}

The gating signals $S_{a}, S_{b}$ and $S_{c}$ determine the switching states of the converter as follows

$$
\begin{aligned}
& S_{a}= \begin{cases}1, & \text { if } S_{1 i} \text { is on and } S_{4 i} \text { is off } \\
0, & \text { if } S_{1 i} \text { is off and } S_{4 i} \text { is on }\end{cases} \\
& S_{b}= \begin{cases}1, & \text { if } S_{3 i} \text { is on and } S_{6 i} \text { is off } \\
0, & \text { if } S_{3 i} \text { is off and } S_{6 i} \text { is on }\end{cases} \\
& S_{c}= \begin{cases}1, & \text { if } S_{5 i} \text { is on and } S_{2 i} \text { is off } \\
0, & \text { if } S_{5 i} \text { is off and } S_{2 i} \text { is on }\end{cases}
\end{aligned}
$$

The vectorial presentation of the switching state is as follows

$$
S=\frac{2}{3}\left(S_{a}+\omega S_{b}+\omega^{2} S_{c}\right)
$$

where $\omega=\mathrm{e}^{j(2 \pi / 3)}$. The output voltage space vectors generated by the inverter are as below

$$
v=\frac{2}{3}\left(V_{a \mathrm{n}}+\omega V_{b \mathrm{n}}+\omega^{2} V_{c \mathrm{n}}\right)
$$

where $V_{a \mathrm{n}}, V_{b \mathrm{n}}$ and $V_{c \mathrm{n}}$ are the phase-to-neutral voltages of the power converter. The voltage vector for the output load is a function of switching states and supply DC voltage as follows

$$
v=S V_{\mathrm{dc}}
$$

\begin{tabular}{|c|c|c|c|c|c|c|c|c|c|c|}
\hline Switching states & $S_{1 i}$ & $S_{2 i}$ & $S_{3 i}$ & $S_{4 i}$ & $S_{5 i}$ & $S_{6 i}$ & $V_{a b}$ & $V_{b c}$ & $V_{c a}$ & $I_{\mathrm{dc}}$ \\
\hline 1 & 1 & 1 & 0 & 0 & 0 & 1 & $V_{\mathrm{dc}}$ & 0 & $-V_{\mathrm{dc}}$ & \\
\hline 2 & 1 & 1 & 1 & 0 & 0 & 0 & 0 & $V_{\mathrm{dc}}$ & $-V_{\mathrm{dc}}$ & \\
\hline 3 & 0 & 1 & 1 & 1 & 0 & 0 & $-V_{\mathrm{dd}}$ & $V_{\mathrm{dc}}$ & 0 & h \\
\hline 4 & 0 & 0 & 1 & 1 & 1 & 0 & $-V_{\mathrm{dc}}$ & 0 & $V_{\mathrm{dc}}$ & $i^{p}+$ \\
\hline 5 & 0 & 0 & 0 & 1 & 1 & 1 & 0 & $-V_{\mathrm{dc}}$ & $V_{\mathrm{dc}}$ & $i$ \\
\hline 6 & 1 & 0 & 0 & 0 & 1 & 1 & $V_{\mathrm{dc}}$ & $-V_{\mathrm{dc}}^{\mathrm{dc}}$ & 0 & $\vec{r}+i$ \\
\hline 7 & 1 & 0 & 1 & 0 & 1 & 0 & 0 & 0 & 0 & 0 \\
\hline 8 & 0 & 1 & 0 & 1 & 0 & 1 & 0 & 0 & 0 & 0 \\
\hline
\end{tabular}

The possible eight switching states and consequently eight voltage vectors are obtained by considering all the possible combinations of the gating signals $S_{a}, S_{b}$ and $S_{c}$.

Table 1 Valid switching states of three-phase VSI 


\subsection{Inductive load model}

The load current of the converter can be presented with space vector as below

$$
i=\frac{2}{3}\left(i^{a}+\omega i^{b}+\omega^{2} i^{c}\right)
$$

Applying the KVL in the power circuit and using the dynamic behaviour of the load current the vector differential equation can be obtained as below

$$
v=R i+L \frac{\mathrm{d} i}{\mathrm{~d} t}
$$

where $v$ is the voltage generated by the inverter, $R$ is the load resistance and $L$ defines the load inductance.

\section{Classical control philosophy}

\subsection{Carrier-based pulse-width modulation (PWM)} control

A carrier-based PWM control scheme is presented in Fig. $2 a$. In this control, PI controller input is generated from the error between the measured load current and the reference current. PI controller is used to set the reference voltages which are compared with fixed carrier signal. The comparator selects the switching states to actuate the converter. In this control, fixed switching frequency is obtained by the carrier signal. The PI controller parameters and the reference current frequency are the main design consideration issues and the system performance depends on it. In PI controller, zero steady-state error for continuous reference is assured and it can present such an error for sinusoidal references. This error proportionally increases with the reference current frequency and may cause system unstable.

\subsection{Hysteresis current control}

The method of hysteresis current control is depicted in Fig. $2 b$. In this control, the reference current and the measured current are compared using hysteresis comparators and determine the switching signals for firing each legs of the converter and forced the load current to remain inside the defined hysteresis band. The performance of the hysteresis control is good with fast dynamic responses. This control is very simple and no need of complex circuits and processors to implement but it has some limitations also. The current error may become beyond to hysteresis band because of the phase interactions. Depending on the operating behaviour and load parameters, the switching frequency may change which causes the resonance effect. Also, at lower power level this control scheme is not suitable enough because of noticeable switching losses. The demerits stated above may prohibited this control in some applications.

\section{Model predictive control}

The proposed MPC control scheme maintains the predictive values closed to their respective references at the end of the sampling instant. The predictive current and selection criterias of voltage vector are explained in below.

\subsection{Discrete-time-model predictive current}

Applying the first-order approximation for derivatives along with the first-order nature of the state equation

$$
\frac{\mathrm{d} i}{\mathrm{~d} t} \cong \frac{i(k+1)-i(k)}{T_{\mathrm{s}}}
$$

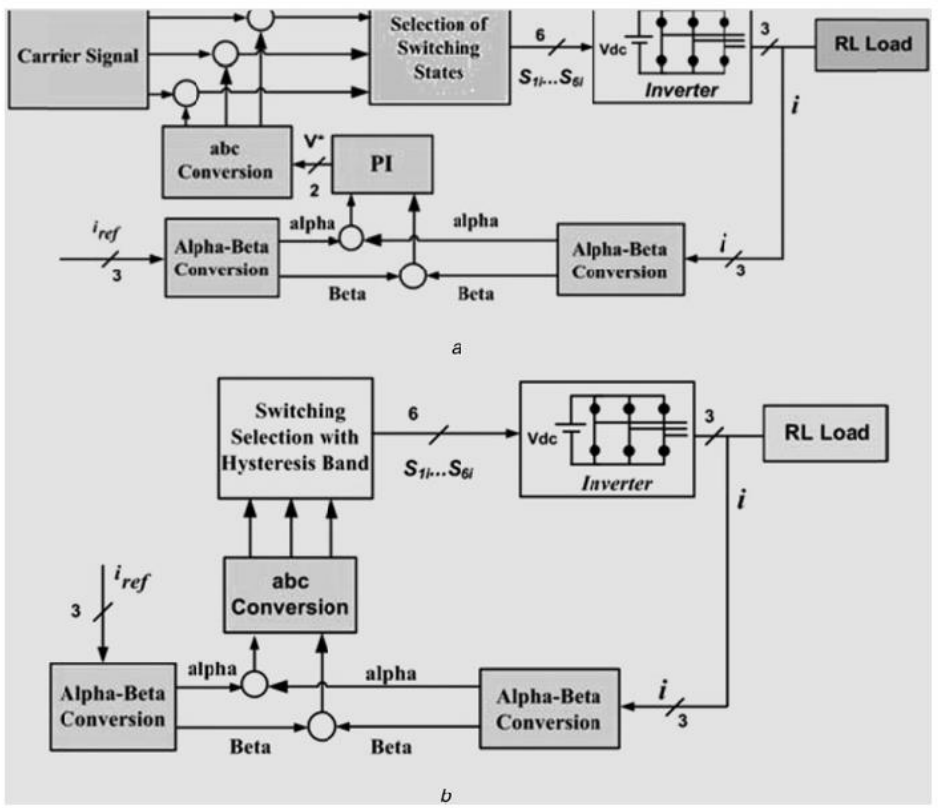

Fig. 2 Classical control methods

$a$ PWM

$b$ Hysteresis current control 
Considering (9) the predictive current becomes

$$
i(k+1)=\frac{T_{\mathrm{s}}}{L} v(k)+i(k)\left(1-\frac{R T_{\mathrm{s}}}{L}\right)
$$

where $i(k)$ is the $k$ th instant load current and $i(k+1)$ is the predictive current.

\subsection{Discrete-time-model predictive torque and flux}

Similarly, with the first-order approximation, stator flux and rotor flux of IM are as below

$$
\begin{gathered}
\psi_{\mathrm{s}}(k)=\psi_{\mathrm{s}}(k-1)+V(k) T_{\mathrm{s}}-R_{\mathrm{s}} i(k) T_{\mathrm{s}} \\
\psi_{\mathrm{r}}(k)=i(k)\left(L_{\mathrm{m}}-\frac{L_{\mathrm{s}} L_{\mathrm{r}}}{L_{\mathrm{m}}}\right)+\frac{L_{\mathrm{r}}}{L_{\mathrm{m}}} \psi_{\mathrm{s}}(k)
\end{gathered}
$$

Predictive stator flux, current and torque becomes

$$
\begin{gathered}
\psi_{\mathrm{s}}(k+1)=\psi_{\mathrm{s}}(k)+V(k) T_{\mathrm{s}}-R_{\mathrm{s}} i(k) T_{\mathrm{s}} \\
i_{\mathrm{s}}(k+1)=\left(1+\frac{T_{\mathrm{s}}}{\tau_{\sigma}}\right) i(k)+\frac{T_{\mathrm{s}}}{\tau_{\sigma}+T_{\mathrm{s}}} \\
\times\left[\frac{1}{R_{\sigma}}\left\{\left(\frac{k_{\mathrm{r}}}{\tau_{\mathrm{r}}}-k_{\mathrm{r}} j \omega_{\mathrm{m}}\right) \psi_{\mathrm{r}}(k)+v(k)\right\}\right] \\
T_{e}(k+1)=\frac{3}{2} p\left\{\psi_{\mathrm{s}}(k+1) \times i(k+1)\right\}
\end{gathered}
$$

\subsection{Cost function calculation}

In MPC algorithm, to express the cost function a positive error is considered for computational simplicity as below

$$
g=\left|i_{\alpha}(k+1)-i_{\text {ref }}^{\alpha}\right|+\left|i_{\beta}(k+1)-i_{\text {ref }}^{\beta}\right|
$$

where $i_{\alpha}(k+1)$ and $i_{\beta}(k+1)$ are the real and imaginary part of the predictive currents; $i_{\text {ref }}^{\alpha}$ is real and $i_{\text {ref }}^{\beta}$ is the imaginary part of the reference currents.

Similarly, the cost function in predictive torque and flux control for IM can be presented as below

$$
g_{\mathrm{IM}}=\left|T_{e}(k+1)-T_{\text {nom }}\right|+\lambda^{*}\left|\psi_{\mathrm{s}}(k+1)-\psi_{\text {ref }}\right|
$$

where $T_{\text {nom }}$ and $\psi_{\text {ref }}$ are the nominal torque and reference flux, respectively; $\psi_{s}(k+1)$ and $T_{e}(k+1)$ are the predictive flux and predictive torque; $\lambda$ is the weighting factor and represent the flux control has an importance rather than the torque control.

\subsection{Predictive control scheme}

The overall control scheme for the MPC current control is given in Fig. $3 a$. The proposed MPC algorithm for current control and IM torque and flux control are presented in Figs. $3 b$ and $c$, respectively. The proposed predictive controller for the predictive current control satisfies the following steps:

(1) Three-phase load current $i(k)$ are measured for the $k$ th sampling time $\left(T_{\mathrm{s}}\right)$ instant.

(2) Three-phase reference current $i_{\text {ref }}$ are at a given values.

(3) Predictive current $i(k+1)$ are predicted for eight of each valid switching states of the converter in the next sampling time period, $T_{\mathrm{s}}(k+1)$.
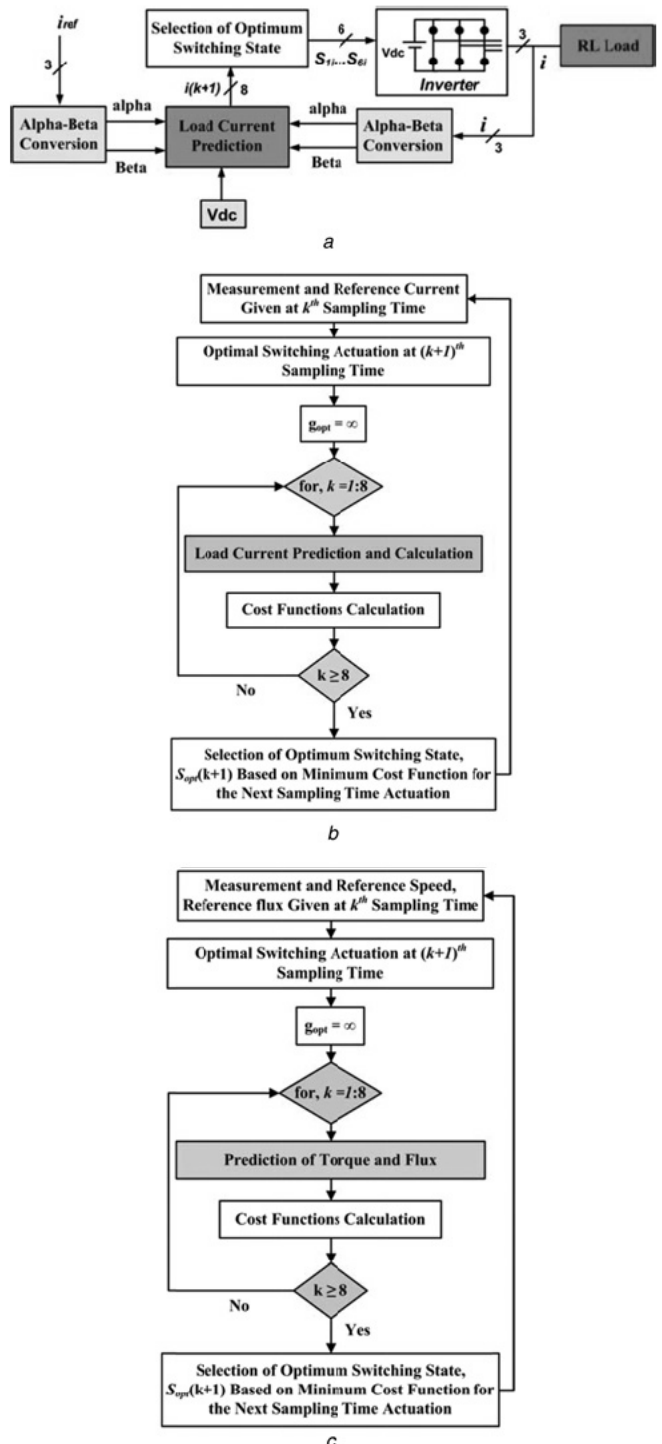

Fig. 3 Predictive control method for RL and IM loads

$a$ Current control scheme

$b$ Current control algorithm

$c$ Torque and flux control algorithm

(4) Predictive values are compared with their respective references and determine the cost functions for all the possible switching states. The switching state corresponds to the minimum cost function value and this switching state is selected to actuate the converter in the next sampling time period.

\section{Results and discussion}

The carrier-based PWM, hysteresis control and predictive current control are verified in MATLAB Simulink as well 
as experimentation in the dSPACE RTI1104 to justify the performance of the proposed MPC scheme. Also, predictive torque and flux control of IM with the simulation studies has been verified in this paper.

\subsection{Simulation results}

The parameters used in the simulations are: DC supply voltage, $V_{\mathrm{dc}}=75 \mathrm{~V}$; load resistance, $R=25 \Omega$ and load inductance, $L=50 \mathrm{mH}$. A carrier frequency of $5 \mathrm{kHz}$ for carrier-based PWM, hysteresis band of $\delta= \pm 0.07$ for hysteresis control and sampling time, $T_{\mathrm{s}}=25 \mu \mathrm{s}$ for the proposed MPC method have been used in the simulations with reference current magnitude of $1 \mathrm{~A}$ and $50 \mathrm{~Hz}$ frequency. A comparison of the proposed MPC control with the classical methods is presented in Figs. 4a-d; Figs. $5 a-d$ and in Figs. $6 a-d$ in consideration with reference tracking and THD $\%$. Figs. $4 a, 5 a$ and $6 a$ present the three-phase measured load current with respect to the references in the verifications of PWM, hysteresis control and predictive control, respectively, with the magnitude of $1 \mathrm{~A}$. Among the results, the PWM load currents are mostly distorted whereas hysteresis control performs slight improvement of distortion but MPC control significantly improve the sinusoidal load current with lower harmonic distortion and performs efficient reference tracking. The output currents are maintained $20 \mathrm{~ms}$ for each cycles and $50 \mathrm{~Hz}$ frequency. The tracking responses of measured $\alpha$-component and $\beta$-component corresponding to the respective references are presented in Figs. $4 b, 5 b$ and $6 b$ for all the control methods, respectively. The results show that, MPC control perform the best among all other classical controls in terms of power quality with lower harmonics and efficient reference tracking.

To show the THD \% comparison, fast Fourier transform (FFT) analysis have been given with the same scale based on fundamental signals and are presented up to 50 order of harmonic in Figs. $4 c, 5 c$ and $6 c$ for all the controls. The THD $\%$ associated with the PWM is $5.52 \%$ in Fig. $4 c$ where 7th, 17th, 19th, 23th and 31th order harmonics highly dominate the current and most of the other harmonics effects on the load current are concentrated within the 13th31 th order of harmonics. For hysteresis control, the THD \% is $3.89 \%$ and is presented in Fig. 5c. In hysteresis control, the dominating harmonics are at 27-33th order. Furthermore, the THD $\%$ for the MPC control method is only $1.49 \%$ presented in Fig. $6 c$. It shows the negligible magnitude of the harmonic order compare to the fundamental signal and no dominant order of harmonic are existed in the current. The phase-to-phase voltages $V_{a b}, V_{b c}$ and $V_{c a}$ are presented in Fig. $6 d$ and very similar to the voltages generated with the classical control philosophies describes in Figs. $4 d$ and $5 d$ with a magnitude of $75 \mathrm{~V}$. The response of the proposed MPC control is very fast as well as best dynamic behaviour with transient response and improved power quality with respect to the classical control methods.

The proposed MPC can be applied for IM load also to validate the performance of the control algorithm. The results associated with the IM are presented in Figs. $7 a-\mathrm{c}$. The parameters used in this simulation are given in Table 2 . In this case, stator reference flux has been assumed as $1 \mathrm{~Wb}$. The IM starts at $0.01 \mathrm{~s}$ without any load torque, varying the reference speed from 0 to $75 \mathrm{rad} / \mathrm{s}$ and the torque is limited to $6.5 \mathrm{Nm}$. At time of $0.01 \mathrm{~s}$, the IM develops $6.5 \mathrm{Nm}$ torque to attain the reference speed of $75 \mathrm{rad} / \mathrm{s}$. The speed controller develop minimum nominal

282

(C) The Institution of Engineering and Technology 2015
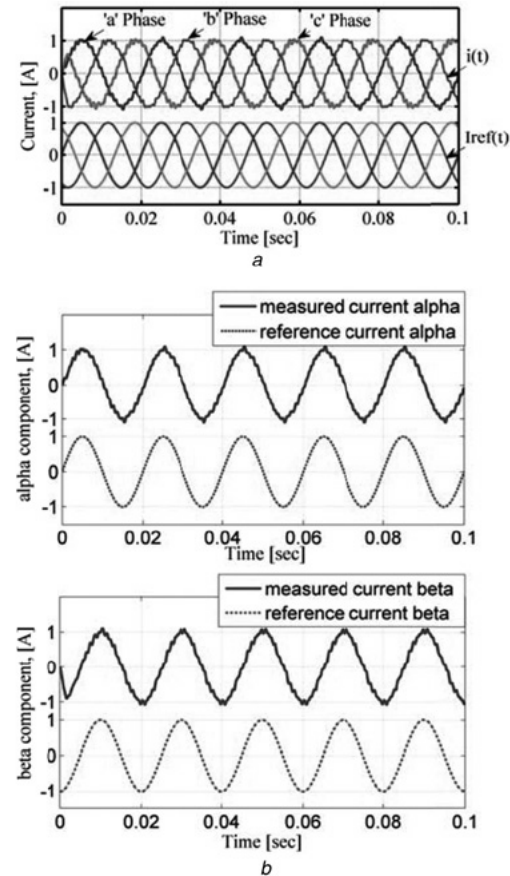

b
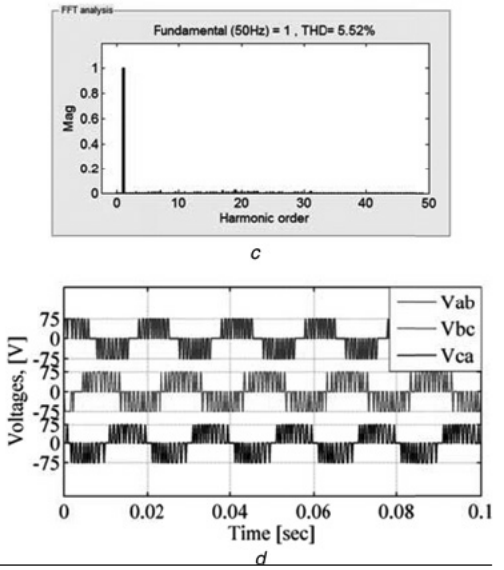

Fig. 4 Simulation results with PWM control

$a$ Three-phase measured current, $i$ [A] (upper) against reference current, $i_{\text {ref }}[\mathrm{A}]$ (lower)

$b \quad \alpha$-component (top): measured current (upper) against reference current (lower)

$\beta$-component (bottom): measured current (upper) against reference current (lower)

$c$ FFT analysis for THD $\%$

$d$ Phase-to-phase voltages $\left(V_{a b}, V_{b c}\right.$ and $\left.V_{c a}\right)$ [V]

torque at full speed and predictive torque follows the nominal torque precisely in Fig. $7 a$. A load torque of $2 \mathrm{Nm}$ has been applied at time of $0.65 \mathrm{~s}$. The motor develop $2 \mathrm{Nm}$ torque at $0.65 \mathrm{~s}$ to mitigate the load demand. The corresponding three phase stator current of IM is given in Fig. $7 b$. The result shows that the IM draws $2.59 \mathrm{~A}$ of current to develop the full torque and consequently the

IET Power Electron., 2015, Vol. 8, Iss. 2, pp. 278-287 doi: $10.1049 /$ iet-pel.2014.0368 


\section{Link to Full-Text Articles :}

http://digital-library.theiet.org/content/journals/10.1049/iet-pel.2014.0368 